\title{
Cortical Axonal Secretion of BDNF in the Striatum Is Disrupted in the Mutant-huntingtin Knock-in Mouse Model of Huntington's Disease
}

\author{
Hyungju Park ${ }^{1,2 *}$ \\ ${ }^{1}$ Division of Neurobiology, Department of Molecular and Cell Biology, Helen Wills Neuroscience Institute, University of \\ California, Berkeley, CA 94720, USA, ${ }^{2}$ Molecular Neurobiology Lab, Department of Structure and Function of Neural \\ Network, Korea Brain Research Institute, Daegu 41062, Korea
}

Deficient BDNF signaling is known to be involved in neurodegenerative diseases such as Huntingtons disease (HD). Mutant huntingtin (mhtt)-mediated disruption of either BDNF transcription or transport is thought to be a factor contributing to striatal atrophy in the HD brain. Whether and how activity-dependent BDNF secretion is affected by the mhtt remains unclear. In the present study, I provide evidence for differential effects of the mhtt on cortical BDNF secretion in the striatum during HD progression. By two-photon imaging of fluorescent BDNF sensor (BDNF-pHluorin and -EGFP) in acute striatal slices of HD knock-in model mice, I found deficient cortical BDNF secretion regardless of the HD onset, but antisense oligonucleotide (ASO)-mediated reduction of htts only rescues BDNF secretion in the early HD brain before the disease onset. Although secretion modes of individual BDNFcontaining vesicle were not altered in the pre-symptomatic brain, the full-fusion and partial-fusion modes of BDNF-containing vesicles were significantly altered after the onset of HD symptoms. Thus, besides abnormal BDNF transcription and transport, our results suggest that mhtt-mediated alteration in activity-dependent BDNF secretion at corticostriatal synapses also contributes to the development of HD.

Key words: BDNF, Huntington's disease, antisense oligonucleotide, corticostriatal synapse

\section{INTRODUCTION}

Huntington's Disease (HD) is a neurodegenerative disorder caused by the mutation of the huntingtin gene ( $h t t)$, which is autosomal dominant [1]. The age of the HD onset varies inversely with the length of CAG repeat expansion in the htt, normally at midlife

Received April 19,2018, Revised May 23,2018,

Accepted May 25, 2018

* To whom correspondence should be addressed. TEL: 82-53-980-8450, FAX: 82-53-980-8339 e-mail:phj2@kbri.re.kr
[2-4]. Typical symptoms include motor dysfunction, cognitive decline, and psychiatric disorders, all of which are believed to be caused by mutant huntingtin (mhtt)-dependent degeneration of many brain areas including the cortex and striatum.

Neurotrophin signaling is known to have a protective function in HD by promoting striatal neuronal survival $[5,6]$. Deficiency in brain derived-neurotrophic factor (BDNF) is linked to diverse brain dysfunctions, and the close relationship between HD pathology and BDNF loss has been extensively demonstrated [58]. In order to reverse the lowered striatal BDNF level and striatal atrophy in the HD brain, various manipulations for increasing BDNF signaling have been found to enhance cell survival and al- 
leviate HD symptoms in HD mouse models. One of approaches to recover the striatal BDNF level is overexpressing $b d n f$ gene in the striatum of HD brain [9-13]. However, there is no direct evidence that overexpressed BDNF could be efficiently secreted in the HD brain. Under the normal condition, cortical BDNF is transported to the striatum anterogradely $[9,14,15]$, and both cellular transport and secretion of BDNF are activity-dependent [16, 17]. Therefore, understanding how BDNF transport and activitydependent secretion is regulated at corticostriatal synapses under normal and diseased states is important for designing BDNFrelated strategies for HD therapy.

In this study, I have utilized a fluorescent BDNF sensor, that is expressed by a viral vector encoding $B d n f$ gene conjugated with $\mathrm{pH}$-sensitive or enhanced green fluorescent protein (pHluorin and EGFP, respectively), to examine the spatiotemporal pattern of BDNF secretion in freshly isolated brain slices $[17,18]$. Heterozygous knock-in mice model for HD were used because they are known to most closely reflect the genetic mutation that is found in human HD patients $[19,20]$. The present study revealed a selective role of mhtts in an impairment of BDNF secretion and suggests a novel therapeutic strategy for preventing deficiency of BDNF secretion in the HD brain by lowering the mhtt level before the onset of disease phenotypes.

\section{MATERIALS AND METHODS}

\section{Animals}

Animal protocols were approved by the Animal Care and Use Committee of University of California, Berkeley. All mice were purchased from Jackson Laboratory except the Q140 and Q175 mice, both of which were provided by the CHDI Foundation and shipped via Jackson Laboratory. Emx1-Cre heterozygotes (genetic background of C57BL/6; Emx $1^{\text {tml(cre)Krj } / J) ~ w e r e ~ c r o s s e d ~ w i t h ~ Q 140 ~}$

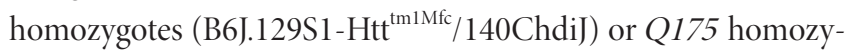
gotes (B6J.129S1-Htt $\left.{ }^{\text {tmlMfc}} / 190 \mathrm{ChdiJ}\right)$ to produce Emx1-Cre/Q140 or Emx1-Cre/Q175 heterozygotes. As a negative control, Emx 1Cre heterozygotes were used.

\section{Expression of BDNF sensor}

AAV-DIO-BDNF-pHluorin was used as described previously [17]. AAV-DIO-BDNF-EGFP was constructed by replacing the pHluorin fragment into the PCR-amplified EGFP fragment. Sequencing and restriction enzyme reactions were performed to verify the plasmid. Custom packaging and purification of both BDNF sensors were performed through UNC Vector Core. 500 $\mathrm{nl}$ (per each hemisphere) of AAV-packaged BDNF sensors were bilaterally injected into the M1 of Emx1-Cre or Emx1-Cre/Q140
(Q175) heterozygote.

\section{Antisense oligonucleotide (ASO) injection}

Injection of $700 \mu \mathrm{g}$ of ASO (in 200 500 $\mathrm{nl}$ of PBS) targeting htt $[21,22]$, which was provided by ISIS (now IONIS) pharmaceutical via the exclusive material transfer agreement (MTA) among the UC Berkeley, CHDI, and ISIS pharmaceutical, was performed in the right lateral ventricle of Q175 mice. As a control, the same volume of PBS was injected. One week later a single bolus ASO or PBS injection, the same mice were again injected with AAVhSyn-BDNF-EGFP into the motor cortex (M1). After additional three more weeks to achieve full BDNF-EGFP expression in the M1 cortex, striatal slices were then prepared to examine activityinduced changes in BDNF-EGFP fluorescence using a two-photon microscope.

\section{Acute brain slice preparation}

Standard artificial cerebral spinal fluid (ACSF) consisted of (in mM) $130 \mathrm{NaCl}, 3.5 \mathrm{KCl}, 1.25 \mathrm{NaH}_{2} \mathrm{PO}_{4}, 24 \mathrm{NaHCO}_{3}, 2 \mathrm{CaCl}_{2}, 2$ $\mathrm{MgCl}_{2}$, and 10 glucose ( $\mathrm{pH} 7.3$ ). Mice were deeply anesthetized with isoflurane, and then transcardially perfused with $\sim 20 \mathrm{ml}$ of slicing ACSF (ACSF containing $10 \mathrm{mM} \mathrm{Mg}^{2+}$ and $0.5 \mathrm{mM} \mathrm{Ca}^{2+}$ ) before the brain was dissected. Parasagittal striatal slices $(400 \mu \mathrm{m}$ thick) were prepared using a vibratome (Leica) using ice-cold slicing ACSF (below $4^{\circ} \mathrm{C}$ ) and maintained at $30 \sim 32^{\circ} \mathrm{C}$ in normal ACSF for 1 hour before electrophysiological recording or twophoton imaging.

\section{Two-photon laser-scanning microscopy}

Two-photon laser-scanning microscopy was performed using an LSM 510 META/NLO Axioimager system (Zeiss; Molecular Imaging Center at UC Berkeley) equipped with a Spectra-Physics MaiTai HP DeepSee laser (700 to $1,020 \mathrm{~nm}$ ) and 403 waterimmersion infrared objective (NA 0.8). BDNF-pH or BDNFEGFP was excited by the $880 \mathrm{~nm}$ laser. The emission signals of BDNF-pH or BDNF-EGFP were acquired by using 500 550 nm band-pass filter. The field of view $(512 \times 512$ pixels, $0.21 \mathrm{~mm} / \mathrm{pixel}$, $0.8 \mathrm{~ms}$ pixel time) was chosen in the striatal slice where cortical projections remained intact and BDNF-pH or BDNF-EGFP was significantly expressed at synaptic bouton-like structures (1 2 $\mu \mathrm{m})$. Slices were placed in a recording chamber, submerged, and continuously perfused $(2 \sim 3 \mathrm{ml} / \mathrm{min}$ ) with oxygenated ACSF (containing $100 \mu \mathrm{M}$ picrotoxin to isolate the glutamatergic synaptic transmission) at room temperature $\left(20 \sim 25^{\circ} \mathrm{C}\right)$.

To record changes in BDNF-pH or BDNF-EGFP intensity in response to electrical stimulation, I acquired at least 100 consecutive images (at $1 \mathrm{~Hz}$ ) as a baseline, then applied electrical stimula- 
tion (HFS: 4 trains of stimuli spaced at $10 \mathrm{~s}$ intervals, with each train containing bursts of 100 spikes at $100 \mathrm{~Hz}$ ) using a tungsten bipolar electrode (WPI) placed on the cortical layer 6 close to the white matter, and then at least additional 200 images at $1 \mathrm{~Hz}$ after stimulation were taken. In some experiments, iso-osmotic ACSF containing $50 \mathrm{mM} \mathrm{NH}_{4} \mathrm{Cl}$ (pH 7.4) was applied to identify axonal BDNF-pH or BDNF-EGFP at the end of each experiment.

\section{Image analysis}

Images were processed and analyzed with ImageJ software (NIH). I presented data as normalized fluorescence changes $\left(\Delta \mathrm{F}_{\mathrm{t}} / \mathrm{F}_{0}\right)$, in which fluorescence changes $\left(\Delta F_{t}\right)$ at a given time were divided by the baseline fluorescence before stimulation $\left(F_{0}\right)$. Puncta with the amplitude of fluorescence reduction $\geq 2$ standard deviations (SDs) from the baseline fluorescence were categorized as undergoing full fusion, and those showing a fluorescence increase $\geq 2$ SDs from the baseline fluorescence were categorized as undergoing a transient event. The rest of the events were defined as no fusion.

\section{Statistical analysis}

Statistical analyses were performed by using Prism 6.0 software (GraphPad). Unpaired Student's t-test and one-way ANOVA with post-test were used for testing significance between two groups and among three or more groups, respectively.

\section{RESULTS}

\section{Activity-dependent BDNF secretion is selectively impaired at cortical axons in the HD brain}

To test whether activity-dependent secretion of BDNF is altered in the brain of HD model mice, BDNF secretion was monitored with acute striatal slices containing corticostriatal projections as previously described [17], using a knock-in type of HD model mice (HdhQ140). Cre/Q140 hybrid mice were obtained by mating Emx1-Cre transgenic mice with $H d h Q 140$ homozygotes, resulting in heterozygotes carrying a single copy of HdhQ140 and Cre genes (Emx1-Cre Q140/+; Fig. 1A). At least 1-year old (48 weeks) Emx1-Cre Q140/+ or Dlx6-Cre Q140/+ mice were injected with AAV-DIO-BDNF-pHluorin (Fig. 1A, B) and used for experiments because Q140 mice are known to display prominent abnormal HD-like phenotypes at this age [19].

After applying electrical stimulation to cortical axons with the high-frequency stimulation (HFS) protocol (Fig. 1C, inset; see Materials and Methods for detailed information) to trigger activity-dependent BDNF secretion at corticostriatal synapses [17, 23], I found that presynaptic secretion of BDNF from cortical axons was greatly reduced in Q140 mice (Fig. 1C and E). Q140 mice showed the reduced activity-induced change in $\Delta \mathrm{F}_{\mathrm{t}} / \mathrm{F}_{0}$ (averaged over 200 400 sec duration after stimulation) of BDNF-pHluorin (BDNF-pH) fluorescence in cortical axons (cortical BDNF-pH: wild-type $(\mathrm{WT})=-0.49 \pm 0.04$ vs. Q140 $=-0.07 \pm 0.03 ; \mathrm{p}<0.0001$, unpaired t-test). In contrast, there was no significant difference in BDNF secretion from medium spiny neuron (MSN) dendrites of Q140 mice, as compared to that found for WT mice (Fig. 1D and E). Activity-induced changes in $\Delta \mathrm{F}_{\mathrm{t}} / \mathrm{F}_{0}$ of BDNF-pHluorin from MSN dendrites of Q140 mice brain slices were not changed as observed in cortical axonal BDNF-pH (averaged $\Delta \mathrm{F}_{\mathrm{t}} / \mathrm{F}_{0}$ of striatal BDNF-pHluorin over 200 400 sec duration after stimulation: $\mathrm{WT}=-0.24 \pm 0.03$ vs. $\mathrm{Q} 140=-0.19 \pm 0.03 ; \mathrm{p}=0.38$, unpaired $\mathrm{t}$-test $)$. Collectively, these results indicate that cortical not striatal BDNF secretion is largely impaired in the striatum of mice with mhtts.

\section{Fusion modes of BDNF-containing vesicles were differen-} tially regulated in cortical and striatal neurites

Next, to explore the mechanism underlying differential effects of mhtts on BDNF secretion from cortical axons vs. striatal MSN dendrites, I analyzed behaviors of individual BDNF-containing vesicles by performing a population analysis of BDNF-pH puncta, which are indicative of BDNF-pH containing secretory vesicles $[17,24]$. I was able to categorize fluorescence changes in BDNF$\mathrm{pH}$ puncta into three differential secretion modes: partial-, full, and no-fusion mode (Fig. 1F, above; see Materials and Methods for more details) [17].

I found that the major secretion mode of pools of BDNFcontaining vesicles (BDNF-vesicles) in cortical axons and MSN neurites of WT mice was full-fusion (Fig. 1F; the fraction of the

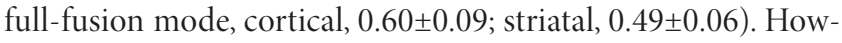
ever, in Q140 mice the major secretion mode of cortical BDNFvesicles was the partial fusion, and I found that an increase in the partial fusion mode of BDNF-vesicles in Q140 mice was statistically significant (Fig. 1F; the fraction of the partial-fusion mode, WT, 0.20 \pm 0.07 ; Q140, 0.52 $\pm 0.09 ; \mathrm{p}<0.05$, unpaired t-test). On the other hand, in Q140 mice striatal BDNF-vesicles rather showed the moderate increase in the fraction of either the full-fusion or partial-fusion mode compared to WT (the fraction of the partialfusion mode: WT BDNF-vesicles $=0.23 \pm 0.07$ vs. Q140 BDNFvesicles $=0.36 \pm 0.02$; the fraction of the full-fusion mode: WT BDNF-vesicles $=0.49 \pm 0.06$ vs. Q140 BDNF-vesicles $=0.59 \pm 0.02$ ), and this was likely due to the significant decrease in the no-fusion mode compared to WT ones (Fig. 1F; the fraction of the no-fusion mode, WT, 0.29 \pm 0.07 ; Q140, 0.05 $\pm 0.01 ; \mathrm{p}<0.05$, unpaired t-test). These results indicate a differential alteration of cortical and striatal mechanisms for activity-dependent BDNF in the HD brain and support the idea that cortical neurons express molecular ma- 
A

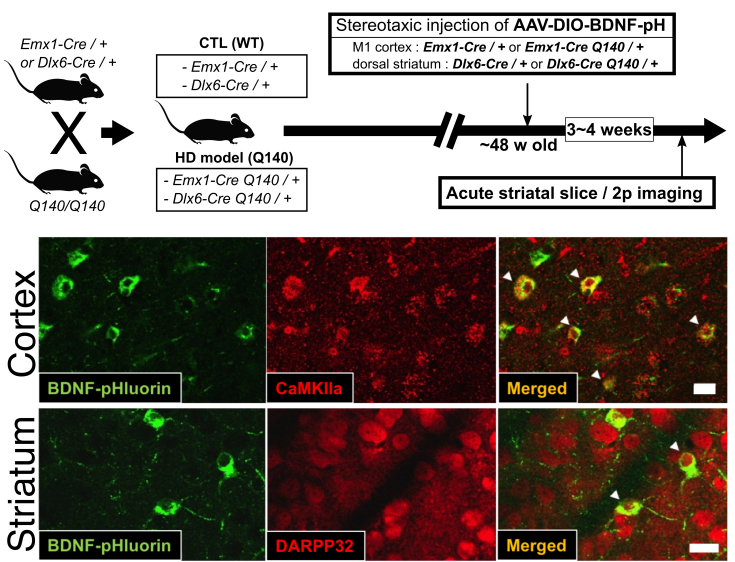

C

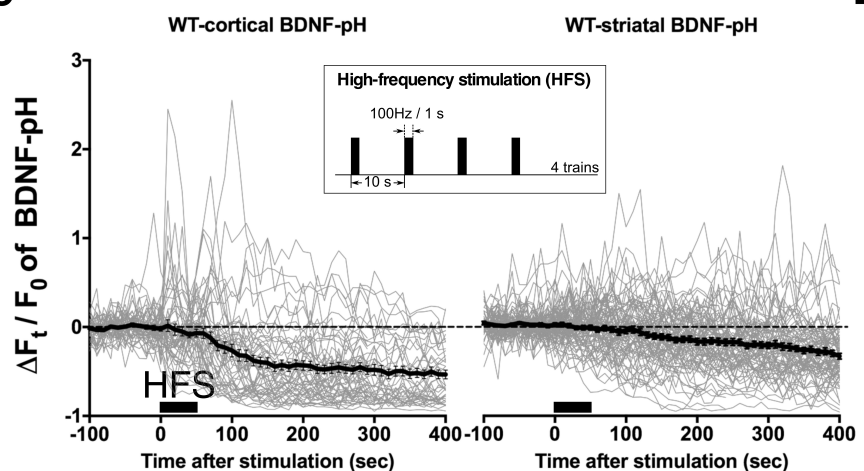

D

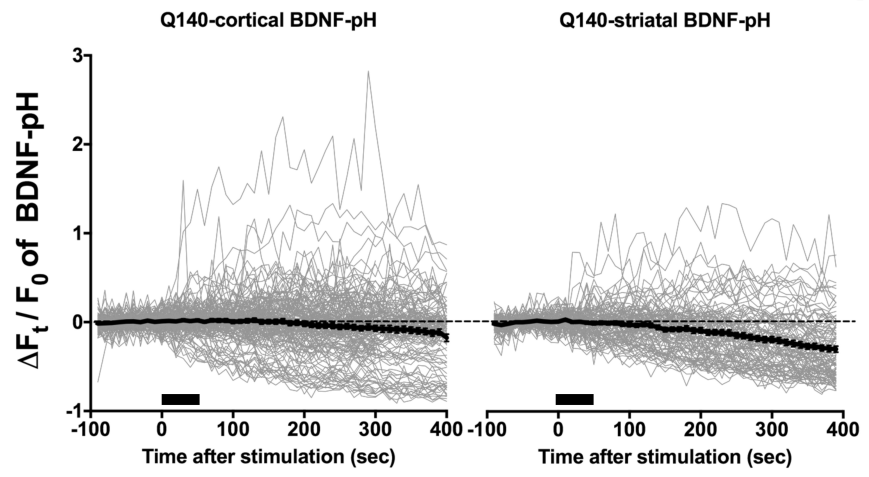

B

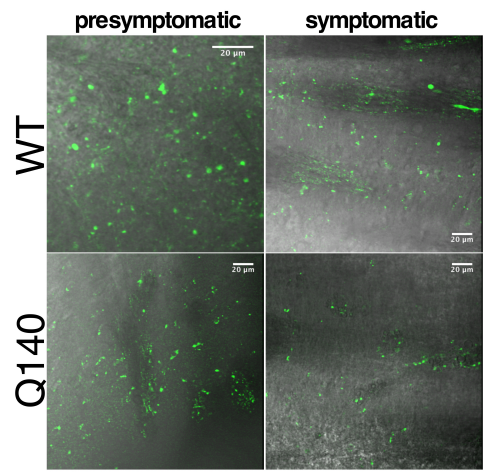

E

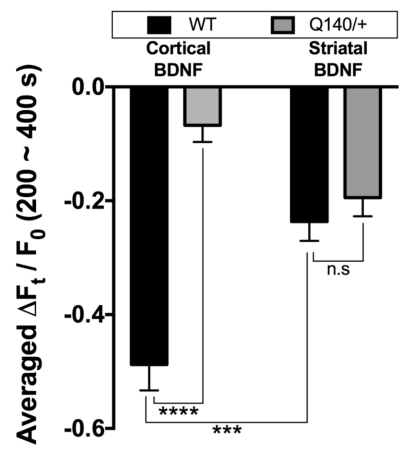

F

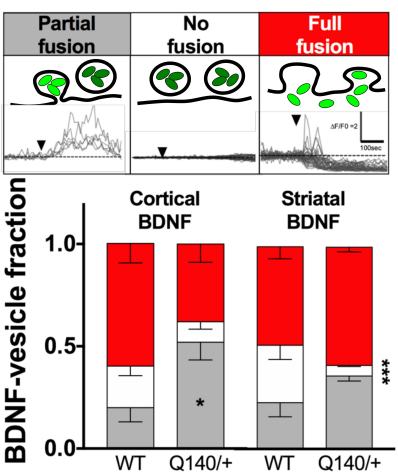

Fig. 1. Differential activity-induced secretion of overexpressed BDNF from cortical axons or striatal neurites in the normal or Q140 heterozygote mice. (A) Above: Schematic diagram depicting experimental conditions including mouse generation, stereotaxic injection of AAV, and two-photon imaging of acute striatal slices. Below: Representative immunohistochemical analysis showing selective BDNF-pH expression in the cortex or dorsal striatum by an injection of AAV-DIO-BDNF-pH into the WT mice (Emx1-Cre/+ or Dlx5/6-Cre/+mice). Note that CamKIIa and DARPP32 are markers for labeling cortical excitatory neuron and striatal medium spiny neurons, respectively. Scale bar $=20 \mu \mathrm{m}$. (B) Representative images demonstrating BDNF-pH puncta signals in the striatum of WT (Emx1-Cre/+) or Q140 (Emx1-Cre Q140/+) mice at 10 weeks (presymptomatic) or 1 year old (symptomatic). AAV-DIOBDNF-pH was injected into the primary motor cortex. (C, D) Two-photon imaging of striatal slices of Emx1-Cre/+ (WT-cortical BDNF-pH) or Dlx5/6Cre/+ (WT-striatal BDNF-pH) mice or Emx1-Cre Q140/+ (Q140-cortical BDNF-pH) or Dlx5-Cre Q140/+ (Q140-striatal BDNF-pH) heterozygote mice (12 24 weeks old) injected with AAV-DIO-BDNF-pH in the primary motor cortex (cortical BDNF-pH) or dorsal striatum (striatal BDNF-pH). 4 times of $100 \mathrm{~Hz}(1 \mathrm{sec}$ ) electrical stimulation (black bar) was given to trigger activity-dependent BDNF secretion. Individual (gray) and averaged (black, mean \pm s. e.m.) traces of BDNF-pH fluorescence intensity measured by two-photon imaging were shown. Inset: Schematic diagram depicting the high-frequency stimulation (HFS) protocol. (E) Bar graphs depict average $\Delta \mathrm{F}_{\mathrm{t}} / \mathrm{F}_{0}$ ( \pm s. e. m.) during 200 400 s after stimulation for all recorded BDNF-pH puncta. Numbers of puncta, at least 3 slices from 2 mice: WT-cortical BDNF-pH, 56; WT-striatal BDNF-pH, 86; Q140-cortical BDNF-pH, 155; Q140-striatal BDNF$\mathrm{pH}, 98 .{ }^{* * *} \mathrm{p}<0,001,{ }^{* * *} \mathrm{p}<0.0001$, one-way ANOVA with Tukey's multiple comparisons test. (F) Above: Categorization of BDNF-containing vesicle fractions according to their changes in fluorescence intensities after electrical stimulation, as "Partial fusion (increased \& recovered fluorescence intensity, gray)," "No fusion (no changes in fluorescence intensity, white), and "Full fusion (decreased fluorescence intensity, red)". Below: Bar graphs depict average fractional changes of individual BDNF-pH puncta (putative BDNF-containing vesicles, mean \pm s.e.m.). ${ }^{\star} \mathrm{p}<0.05$, WT vs. Q140, unpaired t-test. 
chinery that is either essential for BDNF secretion or sensitive to the mhtt.

\section{Differential effects of disease-state on cortical BDNF secretion}

There is evidence for early changes in the striatum in HD model mice and human patients before the onset of motor symptoms [ 19 , 25-27]. It is thus possible that cellular components required for activity-dependent BDNF secretion are already altered before striatal degeneration is observed. To test this idea, I next compared BDNF secretion from cortical axons of 10 week old (presymptomatic age) with that of 1 year old (symptomatic age) Emx1-Cre Q140/+ mice (Fig. 1B). 10 weeks and 1 year old ages were chosen, because Q140 knock-in model mice showed significant htt nuclear staining beginning at 8 weeks, and this htt staining became widespread by 24 weeks of age [19].

Consistent with the finding of impaired BDNF-pH secretion

A

Presymptomatic age (10 weeks old)

Symptomatic age (1 year old)

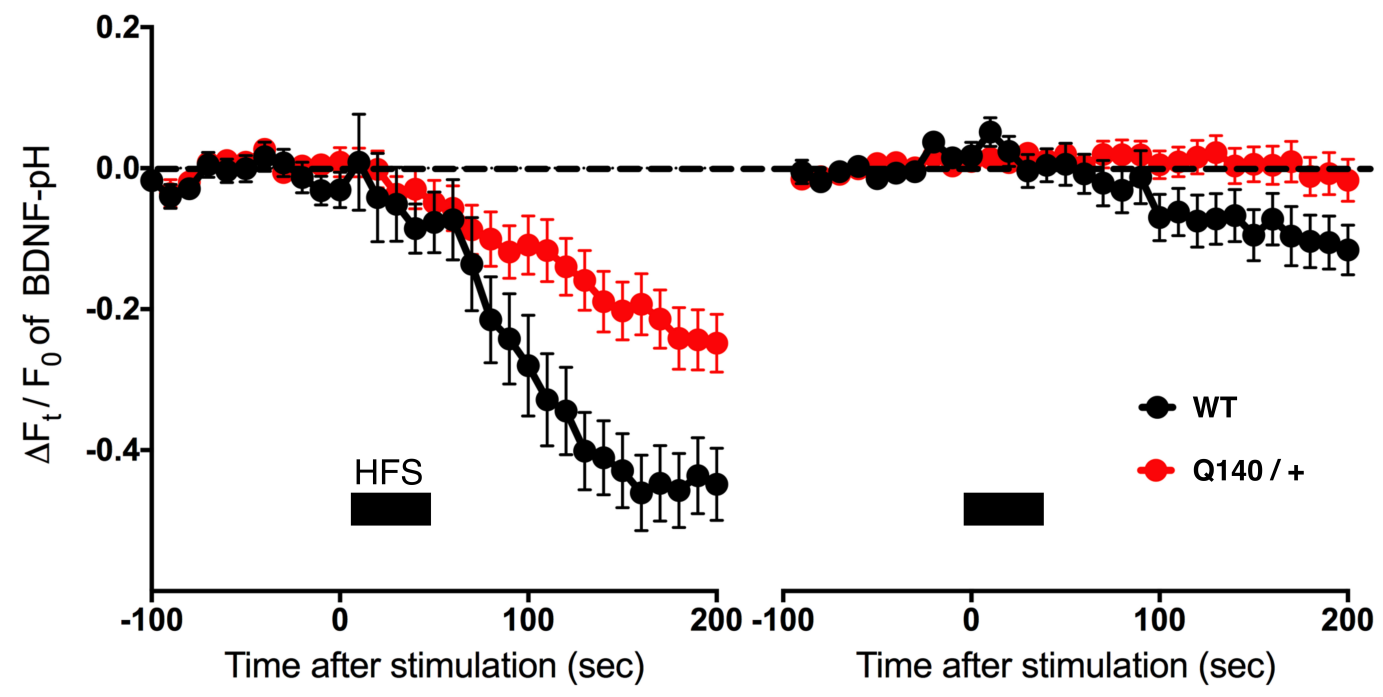

B

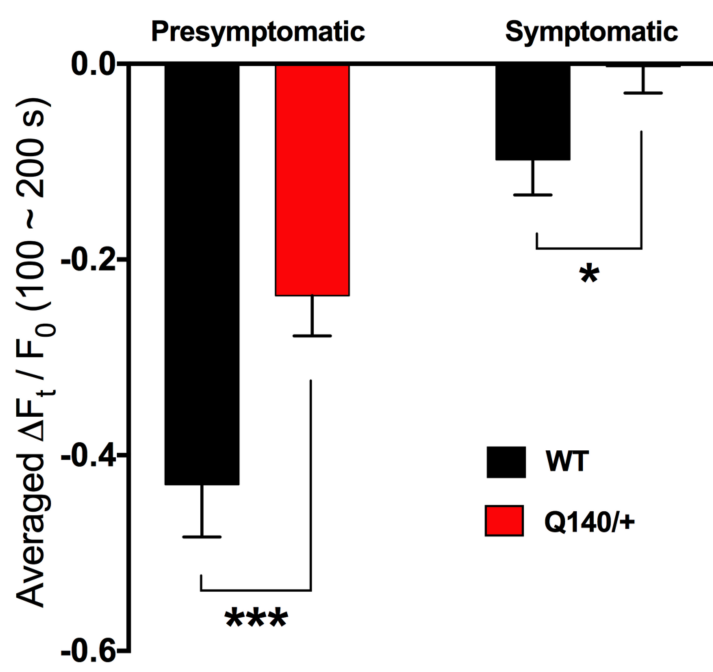

C

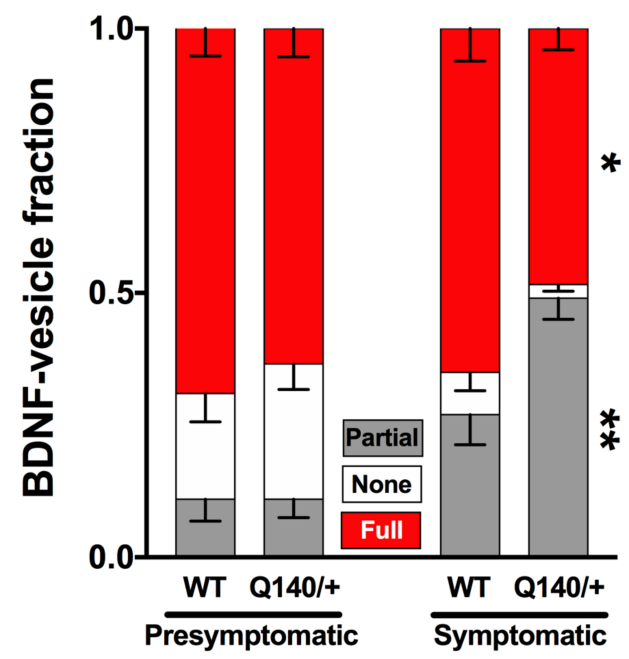

Fig. 2. Disease state-specific alteration of activity-dependent BDNF secretion from cortical axons. (A) Average fluorescence changes with time $\left(\Delta \mathrm{F}_{\mathrm{t}} /\right.$ $\mathrm{F}_{0}$ ) for BDNF-pH puncta of cortical axons in wild-type (Emx1-Cre/+, black) or Q140 (Emx1-Cre Q140/+, red) during the presymptomatic period (10 weeks old) or symptomatic period (1-year-old). Mean \pm s.e.m. HFS (black bar) was given to trigger activity-dependent BDNF secretion. (B) Bar graphs depict average $\Delta \mathrm{F}_{\mathrm{t}} / \mathrm{F}_{0}( \pm$ s. e. $\mathrm{m}$.) during 100 200 s after stimulation for all recorded BDNF-pH puncta. Numbers of puncta from at least 3 slices of 2 mice: presymptomatic wild-type (WT), 56; presymptomatic Q140 (Q140/+), 82; symptomatic wild-type (WT), 62; symptomatic Q140 (Q140/+), 155. ${ }^{*} \mathrm{p}<0,05,{ }^{* * *} \mathrm{p}<0.001$, unpaired t-test. (C) Bar graphs depict average fractional changes of individual BDNF-pH puncta (putative BDNF-containing vesicles, mean \pm s.e.m.). WT vs. Q140: ${ }^{*} \mathrm{p}<0.05,{ }^{* *} \mathrm{p}<0.01$, unpaired t-test. 
from cortical axons in Q140 (Fig. 1), a replicated BDNF secretion assay still showed a significant reduction of HFS-induced BDNF secretion from cortical axons of symptomatic Q140 mice (Fig. 2A and $\mathrm{B}$; averaged $\Delta \mathrm{F}_{\mathrm{t}} / \mathrm{F}_{0}$ over 100 200 sec duration after stimulation, symptomatic age: WT, $-0.10 \pm 0.04 ; \mathrm{Q} 140,-0.00 \pm 0.02 ; \mathrm{p}<0.05$, unpaired t-test). I also found that Q140 mice at the presymptomatic age showed a decrease in cortical BDNF secretion in response to HFS when compared to wild-type mice (Fig. 2A and B; averaged $\Delta \mathrm{F}_{\mathrm{t}} / \mathrm{F}_{0}$ over 100 200 sec duration after stimulation, presymptomatic age: WT, $-0.43 \pm 0.05$; $\mathrm{Q} 140,-0.23 \pm 0.04 ; \mathrm{p}<0.001$, unpaired $\mathrm{t}$ test).

However, a population analysis of BDNF-vesicles in each condition revealed substantial changes in BDNF-vesicle fusion modes with the progress of the disease. At the presymptomatic age, reduced activity-dependent BDNF secretion was not correlated with any change in fusion modes of BDNF-vesicles (Fig. 2C), suggesting that molecular mechanisms involved in activity-dependent cortical BDNF secretion are relatively intact before the HD onset. By contrast, impaired BDNF secretion at the symptomatic age was accompanied by both a significant increase in the partialfusion mode (fraction: WT, 0.27 \pm 0.06 ; Q140, 0.49 $\pm 0.04 ; \mathrm{p}<0.01$, unpaired t-test) and decrease in the full-fusion mode (fraction: $\mathrm{WT}=0.65 \pm 0.06$ vs. $\mathrm{Q} 140=0.48 \pm 0.04 ; \mathrm{p}<0.05$, unpaired $\mathrm{t}$-test $)$, indicating reduced activity-dependent full-fusion of BDNFcontaining vesicles. These results suggest that mhtts not only affect BDNF transcription and transport but also inhibit BDNF secre- tion by disrupting mechanisms for activity-dependent exocytosis of BDNF-containing secretory granules.

\section{ASO-mediated Htt knock-down prevents disruption of axo- nal BDNF secretion in presymptomatic HD brains}

My results demonstrated an association of the mhtt with disrupted activity-dependent BDNF secretion from cortical axons. I next examine whether the mhtt is directly responsible for impaired cortical BDNF secretion in the HD brain, by reducing the htt level with a htt-targeted antisense nucleotide (ASO), which was shown previously to down-regulate mhtt expression [21,22]. Another knock-in HD line with $\sim 180$ CAG repeats in the htt gene (Q175 mice; Emx1-Cre Q175/+ vs. Emx1-Cre/+), which was reported to show faster cortical and striatal atrophies (24 32 weeks old; [20]) than Q140 mice, was used in this experiment. For direct comparison of full-fusion modes of BDNF-vesicles, I utilized BDNFEGFP instead of BDNF-pH, because a decrease in the fluorescent intensity of BDNF-EGFP mostly reflects the full-fusion of BDNFvesicles $[17,18,24]$.

I found that changes in fluorescence intensity of BDNF-EGFP in response to electrical stimulation in all five ASO-injected symptomatic Q175 mice were similar with those observed from PBSinjected Q175 mice (Fig. 3), indicating that ASO-mediated htt knock-down unable to prevent the impairment of BDNF secretion. However, ASO injection to the presymptomatic Q175 (8-week old) mice was effective in preventing defective activity-dependent
A

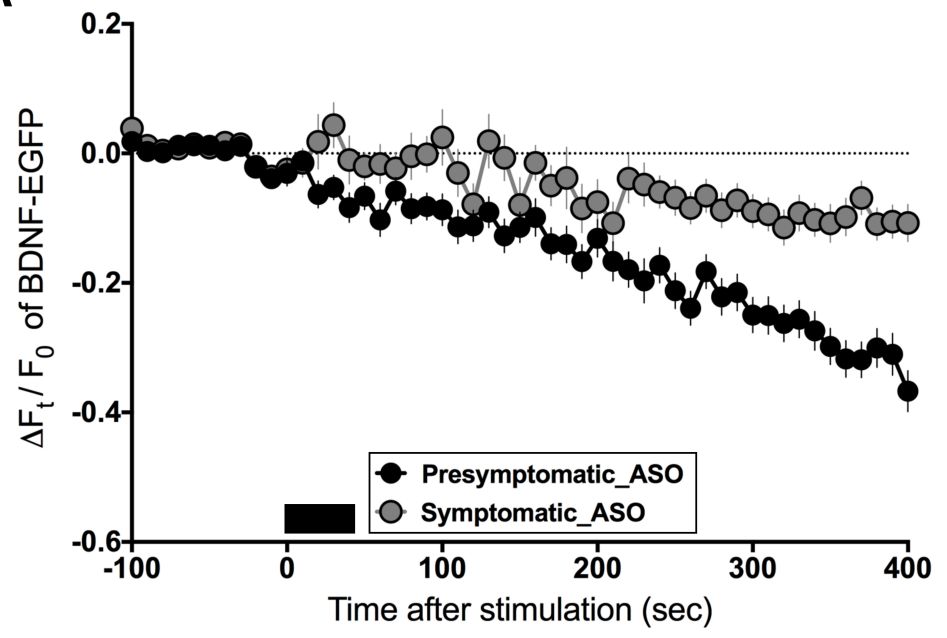

B

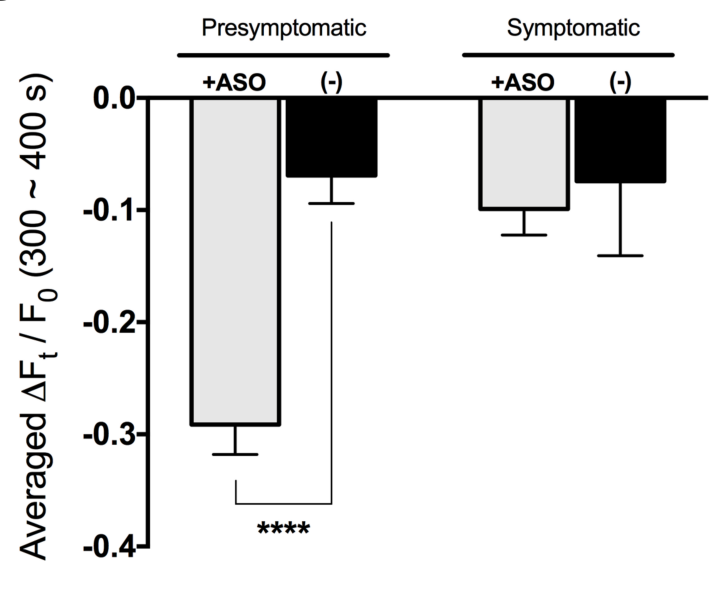

Fig. 3. Reduced Htt level is efficient for alleviating BDNF secretion deficit in the presymptomatic HD brain. (A) Average fluorescence changes with time (mean \pm s. e. m. of $\Delta \mathrm{F}_{\mathrm{t}} / \mathrm{F}_{0}$ ) for BDNF-EGFP puncta of cortical axons in Q175 (Emx1-Cre Q175/+, red) during the presymptomatic period (10 weeks old) or symptomatic period (1-year-old). 4 times of $100 \mathrm{~Hz}$ (1 sec) electrical stimulation (black bar) was given to trigger activity-dependent BDNF secretion. (B) Bar graphs depict average $\Delta \mathrm{F}_{\mathrm{t}} / \mathrm{F}_{0}( \pm$ s. e. m.) during 300 400 s after stimulation for all recorded BDNF-EGFP puncta. Numbers of puncta from at least 3 slices of 3 mice: presymptomatic Q175 with PBS injection (-), 32; presymptomatic Q175 with ASO injection (+ASO), 99; symptomatic Q175 with PBS injection, 29; symptomatic Q175 with ASO injection, $94 .{ }^{*} \mathrm{p}<0,05,{ }^{* * *} \mathrm{p}<0.001$, unpaired t-test. 
BDNF secretion. I found that overall cortical BDNF secretion was significantly recovered in three of five presymptomatic Q175 mice, which were injected with the same amount of htt ASO with that injected in the symptomatic Q175 mice (Fig. 3; ${ }^{* * *} \mathrm{p}<0.001$ ). These results indicate that a mhtt reduction during the early stage of $\mathrm{HD}$ could be beneficial for restoring BDNF level in the striatum through recovering activity-dependent BDNF secretion, although same application was not effective for reversing impaired BDNF secretion in the symptomatic HD brain.

\section{DISCUSSION}

In this study, I show that cortical activity-dependent BDNF secretion is abnormal, but no alteration of striatal BDNF secretion was observed in the brain with HD (Fig. 1E). These results suggest that cortical activity-dependent BDNF secretion is more vulnerable to the mhtt than striatal BDNF secretion from MSNs. Since striatal MSN do not express a significant level of BDNF $[14,15]$, insensitivity of activity-dependent BDNF secretion to the mhtt might result from the lack of molecular machinery essential for BDNF secretion. Overall activity-induced cortical BDNF-pH secretion in WT striatal slices was significantly higher than striatal one by same stimulation (Fig. $1 \mathrm{E} ; \mathrm{p}<0.001$, unpaired $\mathrm{t}$-test), but this is probably because cortical axons directly received electrical stimulation, whereas activity in striatal neurites was evoked by electrical stimulation-induced synaptic transmission. Since pre- synaptic glutamate release at corticostriatal synapses was shown to be already reduced at this HD stage [26, 27], the reduced nofusion mode of striatal BDNF-vesicles in the HD brain might be reminiscent of the increased postsynaptic receptor sensitivity as a compensatory mechanism caused by decreased presynaptic inputs, and this alteration may affect activity-dependent BDNF secretion from striatal neurons of HD brains.

The exact molecular mechanisms of BDNF secretion affected by mhtts are still unclear. Several studies provide molecular evidence for mhtt-dependent alteration of vesicular exocytosis. Overexpression of mutant huntingtin in PC12 cells depleted vesicular machinery such as complexin II, resulting in impaired $\mathrm{Ca}^{2+}$-triggered vesicular exocytosis [28]. Moreover, impaired astrocytic BDNF release in Q140 knock-in mice is reported to be caused by the abnormal function of Rab3a, a small GTPase localized on membranes of dense-core vesicles [29]. Since an ATP release from astrocytic dense-core vesicles was also significantly reduced in Q140 mice [29], it is possible that mhtts disrupt general $\mathrm{Ca}^{2+}$-dependent exocytosis of dense-core vesicles. Despite normal fusion modes of BDNF-vesicles in the presymptomatic HD brain (Fig. 2C), I cannot exclude a possibility that a mhtt reduction reverses a deficient BDNF transport in the presymptomatic HD brain that was found to be present during early developmental stages [6, 7], still capable of increasing the chance of BDNF secretion in the striatum.

My study did not directly show whether the ASO treatment restored impaired secretion of a pro-form (proBDNF) or mature
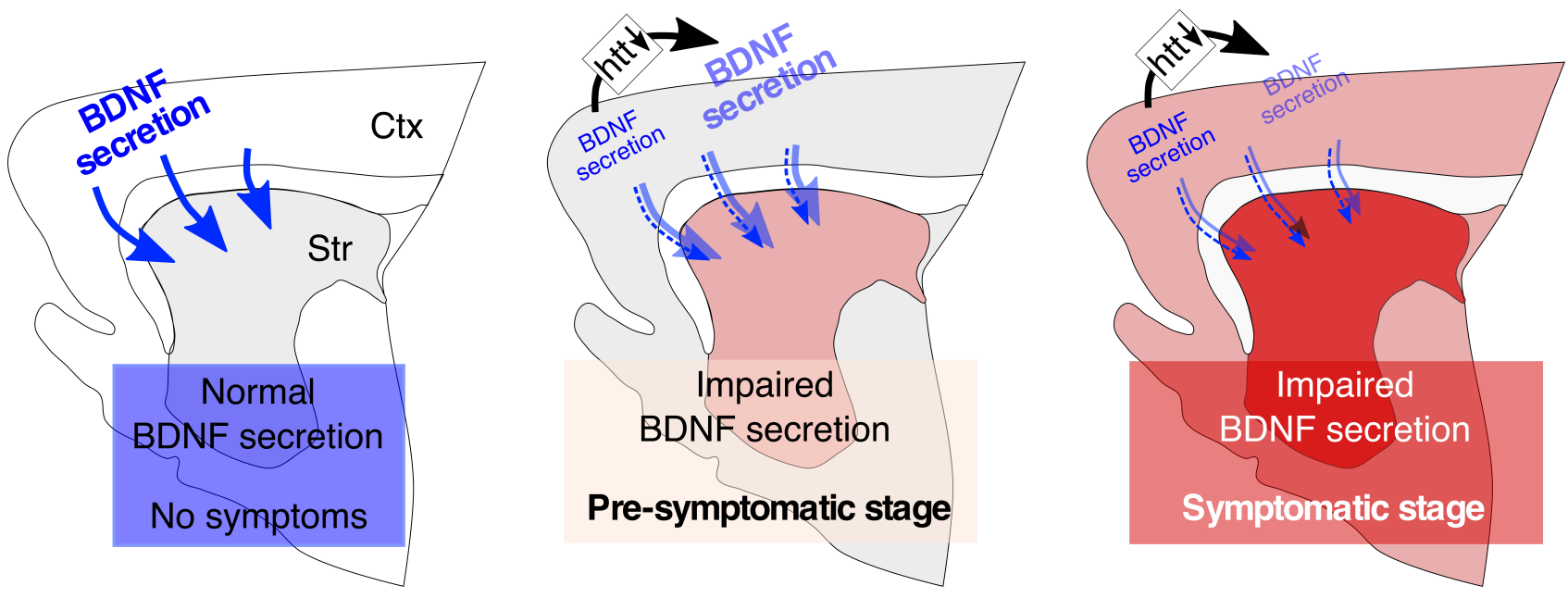

Fig. 4. Proposed model for a disease progress-dependent alteration of activity-dependent BDNF secretion from cortical axons to the striatum. A schematic illustration of cortical presynaptic BDNF secretion in the normal brain or HD brains at the presymptomatic or symptomatic stage. In addition to impaired synthesis and transport of BDNF, I propose that BDNF secretion is also downregulated by mutant huntingtin (htt), probably through altered actions of molecular mechanisms regulating activity-dependent docking or exocytosis of BDNF-containing vesicles. Since mhtt-mediated disruption of BDNF secretion is prominent during the presymptomatic stage of $\mathrm{HD}$, and this could be reversed by selective reduction of mhtt, a combined treatment targeting both the presymptomatic stage of $\mathrm{HD}$ and restoration of BDNF expression could be a promising strategy for reversing striatal degeneration in the HD brain. 
form of BDNF (mature BDNF). A line of evidence suggests that the mature $\mathrm{BDNF}$ is a major form of $\mathrm{BDNF}$ secreted from cortical axons in the striatum. A previous study demonstrated that forebrain overexpression of BDNF in the HD model mice increases striatal TrkB signaling and cell survivals [9], both of which are mediated by mature BDNFs. Moreover, induction of corticostriatal long-term potentiation (LTP) was dependent on mature BDNF secretion from cortical presynaptic axons $[30,31]$ as shown at hippocampal synapses, where LTP and long-term depression (LTD) were selectively regulated by mature and proBDNF signaling, respectively [16, 30-33]. Thus, I suggest that restoration of BDNF signaling by the ASO treatment is mostly mediated by mature BDNFs.

Together, not only providing additional evidence for a mhtt-mediated disruption of BDNF secretion, the present study also points to a combination of the mhtt reduction and BDNF overexpression for prevention of $\mathrm{HD}$ after early detection of the disease (Fig. 4). In addition to a neuronal delivery of BDNF, I expect that glial BDNF overexpression or implantation of genetically modified cells with BDNF expression would also be useful $[10,11,13]$ in promoting striatal BDNF level and reducing HD symptoms, with the efficacy greatly enhanced if accompanied with the mhtt reduction before HD symptoms occur.

\section{ACKNOWLEDGEMENTS}

I would like to thank Dr. Mu-ming Poo for discussion and advices. This work was supported by grants from the CHDI Foundation (CHDI A3794), and KBRI basic research program through Korea Brain Research Institute funded by Ministry of Science and ICT (18-BR-01-03).

\section{REFERENCES}

1. Gusella JF, MacDonald ME (1995) Huntington's disease: CAG genetics expands neurobiology. Curr Opin Neurobiol 5:656-662.

2. MacDonald ME, Barnes G, Srinidhi J, Duyao MP, Ambrose CM, Myers RH, Gray J, Conneally PM, Young A, Penney J (1993) Gametic but not somatic instability of CAG repeat length in Huntington's disease. J Med Genet 30:982-986.

3. Myers RH, MacDonald ME, Koroshetz WJ, Duyao MP, Ambrose CM, Taylor SA, Barnes G, Srinidhi J, Lin CS, Whaley WL, Lazzarini AM, Schwarz M, Wolff G, Bird ED, Vonsattel JP, Gusella JF (1993) De novo expansion of a (CAG)n repeat in sporadic Huntington's disease. Nat Genet 5:168-173.

4. Snell RG, MacMillan JC, Cheadle JP, Fenton I, Lazarou LP,
Davies P, MacDonald ME, Gusella JF, Harper PS, Shaw DJ (1993) Relationship between trinucleotide repeat expansion and phenotypic variation in Huntington's disease. Nat Genet 4:393-397.

5. Baydyuk M, Xu B (2014) BDNF signaling and survival of striatal neurons. Front Cell Neurosci 8:254.

6. Zuccato C, Cattaneo E (2009) Brain-derived neurotrophic factor in neurodegenerative diseases. Nat Rev Neurol 5:311322.

7. Gauthier LR, Charrin BC, Borrell-Pagès M, Dompierre JP, Rangone H, Cordelières FP, De Mey J, MacDonald ME, Lessmann V, Humbert S, Saudou F (2004) Huntingtin controls neurotrophic support and survival of neurons by enhancing BDNF vesicular transport along microtubules. Cell 118:127138.

8. Strand AD, Baquet ZC, Aragaki AK, Holmans P, Yang L, Cleren C, Beal MF, Jones L, Kooperberg C, Olson JM, Jones KR (2007) Expression profiling of Huntington's disease models suggests that brain-derived neurotrophic factor depletion plays a major role in striatal degeneration. J Neurosci 27:11758-11768.

9. Gharami K, Xie Y, An JJ, Tonegawa S, Xu B (2008) Brainderived neurotrophic factor over-expression in the forebrain ameliorates Huntington's disease phenotypes in mice. J Neurochem 105:369-379.

10. Giralt A, Friedman HC, Caneda-Ferrón B, Urbán N, Moreno E, Rubio N, Blanco J, Peterson A, Canals JM, Alberch J (2010) $B D N F$ regulation under GFAP promoter provides engineered astrocytes as a new approach for long-term protection in Huntington's disease. Gene Ther 17:1294-1308.

11. Giralt A, Carretón O, Lao-Peregrin C, Martín ED, Alberch J (2011) Conditional BDNF release under pathological conditions improves Huntington's disease pathology by delaying neuronal dysfunction. Mol Neurodegener 6:71.

12. Xie Y, Hayden MR, Xu B (2010) BDNF overexpression in the forebrain rescues Huntington's disease phenotypes in YAC128 mice. J Neurosci 30:14708-14718.

13. Zimmermann T, Remmers F, Lutz B, Leschik J (2016) ESCderived BDNF-overexpressing neural progenitors differentially promote recovery in Huntington's disease models by enhanced striatal differentiation. Stem Cell Reports 7:693706.

14. Altar CA, Cai N, Bliven T, Juhasz M, Conner JM, Acheson AL, Lindsay RM, Wiegand SJ (1997) Anterograde transport of brain-derived neurotrophic factor and its role in the brain. Nature 389:856-860.

15. Conner JM, Lauterborn JC, Yan Q, Gall CM, Varon S (1997) 
Distribution of brain-derived neurotrophic factor (BDNF) protein and mRNA in the normal adult rat CNS: evidence for anterograde axonal transport. J Neurosci 17:2295-2313.

16. Park H, Poo MM (2013) Neurotrophin regulation of neural circuit development and function. Nat Rev Neurosci 14:7-23.

17. Park H, Popescu A, Poo MM (2014) Essential role of presynaptic NMDA receptors in activity-dependent BDNF secretion and corticostriatal LTP. Neuron 84:1009-1022.

18. Lu H, Park H, Poo MM (2013) Spike-timing-dependent BDNF secretion and synaptic plasticity. Philos Trans R Soc Lond B Biol Sci 369:20130132.

19. Heng MY, Detloff PJ, Albin RL (2008) Rodent genetic models of Huntington disease. Neurobiol Dis 32:1-9.

20. Southwell AL, Smith-Dijak A, Kay C, Sepers M, Villanueva EB, Parsons MP, Xie Y, Anderson L, Felczak B, Waltl S, Ko S, Cheung D, Dal Cengio L, Slama R, Petoukhov E, Raymond LA, Hayden MR (2016) An enhanced Q175 knock-in mouse model of Huntington disease with higher mutant huntingtin levels and accelerated disease phenotypes. Hum Mol Genet 25:3654-3675.

21. Carroll JB, Warby SC, Southwell AL, Doty CN, Greenlee S, Skotte N, Hung G, Bennett CF, Freier SM, Hayden MR (2011) Potent and selective antisense oligonucleotides targeting single-nucleotide polymorphisms in the Huntington disease gene / allele-specific silencing of mutant huntingtin. Mol Ther 19:2178-2185.

22. Southwell AL, Skotte NH, Kordasiewicz HB, Østergaard ME, Watt AT, Carroll JB, Doty CN, Villanueva EB, Petoukhov E, Vaid K, Xie Y, Freier SM, Swayze EE, Seth PP, Bennett CF, Hayden MR (2014) In vivo evaluation of candidate allelespecific mutant huntingtin gene silencing antisense oligonucleotides. Mol Ther 22:2093-2106.

23. Jia Y, Gall CM, Lynch G (2010) Presynaptic BDNF promotes postsynaptic long-term potentiation in the dorsal striatum. J Neurosci 30:14440-14445.

24. Matsuda N, Lu H, Fukata Y, Noritake J, Gao H, Mukherjee S, Nemoto T, Fukata M, Poo MM (2009) Differential activitydependent secretion of brain-derived neurotrophic factor from axon and dendrite. J Neurosci 29:14185-14198.

25. Gusella JF, MacDonald ME, Ambrose CM, Duyao MP (1993) Molecular genetics of Huntington's disease. Arch Neurol 50:1157-1163.

26. Joshi PR, Wu NP, André VM, Cummings DM, Cepeda C, Joyce JA, Carroll JB, Leavitt BR, Hayden MR, Levine MS, Bamford NS (2009) Age-dependent alterations of corticostriatal activity in the YAC128 mouse model of Huntington disease. J Neurosci 29:2414-2427.

27. Deng YP, Wong T, Wan JY, Reiner A (2014) Differential loss of thalamostriatal and corticostriatal input to striatal projection neuron types prior to overt motor symptoms in the Q140 knock-in mouse model of Huntington's disease. Front Syst Neurosci 8:198.

28. Edwardson JM, Wang CT, Gong B, Wyttenbach A, Bai J, Jackson MB, Chapman ER, Morton AJ (2003) Expression of mutant huntingtin blocks exocytosis in PC12 cells by depletion of complexin II. J Biol Chem 278:30849-30853.

29. Hong Y, Zhao T, Li XJ, Li S (2016) Mutant Huntingtin impairs BDNF release from astrocytes by disrupting conversion of Rab3a-GTP into Rab3a-GDP.J Neurosci 36:8790-8801.

30. Korte M, Carroll P, Wolf E, Brem G, Thoenen H, Bonhoeffer $\mathrm{T}$ (1995) Hippocampal long-term potentiation is impaired in mice lacking brain-derived neurotrophic factor. Proc Natl Acad Sci U S A 92:8856-8860.

31. Zakharenko SS, Patterson SL, Dragatsis I, Zeitlin SO, Siegelbaum SA, Kandel ER, Morozov A (2003) Presynaptic BDNF required for a presynaptic but not postsynaptic component of LTP at hippocampal CA1-CA3 synapses. Neuron 39:975990.

32. Pang PT, Teng HK, Zaitsev E, Woo NT, Sakata K, Zhen S, Teng KK, Yung WH, Hempstead BL, Lu B (2004) Cleavage of proBDNF by tPA/plasmin is essential for long-term hippocampal plasticity. Science 306:487-491.

33. Woo NH, Teng HK, Siao CJ, Chiaruttini C, Pang PT, Milner TA, Hempstead BL, Lu B (2005) Activation of p75NTR by proBDNF facilitates hippocampal long-term depression. Nat Neurosci 8:1069-1077. 